\title{
COMMENT
}

\section{Poverty and chronic illness: why safety net programs matter}

\author{
Deborah Bloch (iD) and Ann Chahroudi ${ }^{1,2}$ \\ Pediatric Research (2019) 85:743-744; https://doi.org/10.1038/s41390-019-0363-2
}

Articles in this month's issue of Pediatric Research authored by Kochmanski, Oliveira, and Monthé-Drèze and their respective teams emphasize the importance of weight and nutrition on pediatric health, which here we will discuss in the context of poverty, a far too common social determinant of health. These three articles touch upon different contributors to and outcomes of unhealthy weight in children. In attempting to unravel the contribution of nurture vs. nature in childhood obesity development, Kochmanski, et al. utilizing a cohort from the Michigan Momentum Center Healthy Families Project, highlight the role of epigenetics by age-related DNA methylation at seven genetic sites in children of different age groups. ${ }^{1}$ Oliveira, et al. propose that metabolic syndrome in obese Latino males, more so than severity of obstructive sleep apnea, causes autonomic dysfunction, ${ }^{2}$ and Monthé-Drèze, et al. investigate the relationship between maternal obesity and poor neurodevelopmental outcomes of offspring. ${ }^{3}$ These articles support the need for continued research on modifiable factors to prevent childhood obesity and malnutrition and call for resources to address their root causes, such as poverty, in early life.

It is well established that in both developing and developed countries, unhealthy weight leads to both short term and long term adverse health outcomes. Poverty and unhealthy weight go hand-in-hand. Though they are seemingly dichotomous outcomes, both obesity and malnutrition are often driven by the same factors: poor access to or unaffordability of healthy foods (termed food insecurity). Malnutrition early in life has been linked to chronic illnesses, such as diabetes, hyperlipidemia, and cardiovascular disease in adulthood, which are all also associated with obesity. ${ }^{4}$

According to the 2017 United States Census Bureau, 39.7 million people live under the poverty line. ${ }^{5}$ Approximately one third of these millions are children under the age of 18 years. Poverty disproportionately affects African-American, Hispanic, and American Indian/Alaskan Native children. ${ }^{6}$ Chronic toxic stress, induced by poverty, adversely impacts the hypothalamic-pituitaryadrenocortical axis leading to physiologic dysregulation of cortisol production, increased inflammation, and impaired physical growth and neurodevelopment. Compared to wealthier peers, children living in poverty are more likely to experience tobacco exposure, asthma, hypertension, poor nutrition, untreated dental carries, mental health problems including future substance abuse, and poor academic performance. Mothers living in poverty are more likely to have premature and underweight babies, as well as higher rates of infant mortality. Through a variety of environmental and physiologic mechanisms, the cascading health consequences of poverty and toxic stress have been shown to be epigenetic, thus poverty felt by one generation can have long lasting sequelae for generations to come. ${ }^{6}$ All too often those in the direst health circumstances because of poor social determinants are the ones who have the least access to healthcare, resulting in delayed presentation for treatable illnesses and insufficient benefit from preventative services.

Screening for factors impacting health, such as poverty, food insecurity, housing instability, and exposure to violence, should be integrated into pediatric well and sick visits. We cannot provide comprehensive care for children without identifying and addressing needs of families. Pediatricians provide medical homes, a central place where holistic treatments for the medical and social determinants of health can be accessed. Routine referral of families to nutrition services, such as SNAP (Supplemental Nutrition Assistance Program) and WIC (Special Supplemental Nutrition Program for Women, Infants, and Children) is one of the tools pediatricians use to address poverty and food insecurity. Screening for neurodevelopmental problems and promptly offering therapy through Early Interventions Programs along with facilitating enrollment in Early Head Start and Head Start programs are further resources pediatricians have to address poverty. Finally, it must be noted that medical, surgical, dental, and mental health services for over 35 million children without another source of medical insurance are covered through Medicaid and the Children's Health Insurance Program (CHIP). ${ }^{7}$ Multiple studies have shown the medical and economic benefits of Medicaid. ${ }^{8}$ For example, though healthcare utilization may be high in childhood, Medicaid led to healthier adults as measured by reduced number of hospitalizations at age 25 and better composite health index scores that assessed patients for hypertension, diabetes, heart disease, and obesity.,10 Medicaid saves millions from healthcare related financial hardship and some studies have shown that it improves college attendance and decreases high school dropout. ${ }^{11,12}$ All of these factors help alleviate the burdens of poverty and ensure healthier lives for the next generations. The American Academy of Pediatrics policy statement "Poverty and Child Health in the United States," released in 2016, includes a comprehensive summary of the resources available to pediatricians and families faced with the burden of poverty. ${ }^{13}$ At a time of unpredictable funding and fear regarding continued access to social and medical services, it is clear that support for safety net programs that help children, and the research initiatives to improve them, are crucial for progression to a healthy and prosperous adulthood.

Social determinants of health, including poverty also adversely impact chronic infectious diseases. As an example, new Human Immunodeficiency Virus (HIV) infections, while on the decline for decades in the US, increased among persons aged 25-34 years between 2010-2015, and new diagnoses in 13-24 year olds

\footnotetext{
${ }^{1}$ Department of Pediatrics, Division of Pediatric Infectious Diseases, Emory University School of Medicine, Atlanta, GA, USA and ${ }^{2}$ Ponce de Leon Center Family \& Youth Clinic, Infectious Diseases Program, Grady Health System, Atlanta, GA, USA

Correspondence: Ann Chahroudi (ann.m.chahroudi@emory.edu)
}

Received: 5 February 2019 Accepted: 27 February 2019

Published online: 6 March 2019 
exceeded all groups older than 35 years in 2017. In Georgia, rates of new HIV diagnoses were 24.9 per 100,000 people in 2017, the highest in any state. And in Atlanta, African-American men who have sex with men have a $50 \%$ lifetime risk of becoming infected with HIV. ${ }^{14}$ These increases in infection rates are occurring within the context of the highest rate of poverty in the country, with childhood poverty well above the national average. Lack of implementation of Medicaid expansion across the Southeast has left more individuals uninsured than in any other region. A natural consequence of this lost support is less exposure to preventative care services for uninsured adolescents and young adults, such as safer sex counseling, routine testing for HIV, and other sexually transmitted infections, pre-exposure prophylaxis for HIV, and birth control. Absent the structural provisions created by steady, reliable health insurance, these numbers indicate we are not doing enough to prepare adolescents and young adults to make healthy life choices.

While once considered a death sentence, HIV in children and adolescents is now a treatable chronic illness for patients with access to and engagement in care. The Ryan White Comprehensive AIDS Resources Emergency (CARE) Act, named for a 13 year old boy diagnosed with HIV in 1985 and passed by Congress in 1990 , provides a system for medical care and support services for people living with HIV who are uninsured or underinsured. $62.8 \%$ of clients served by the Ryan White Program in 2017 were living at $\leq 100 \%$ of the federal poverty level. The impact of this essential funding source cannot be underestimated. Approximately $66 \%$ youth aged 13-24 years who are living with HIV are enrolled in Ryan White programs across the country. Additionally, the AIDS Drug Assistance Program (ADAP) provides states with resources to distribute HIV medications and can be credited with serving over 230,000 individuals. We note that parents living with HIV routinely utilize services through the Ryan White Program and ADAP, thereby also providing benefit to their children, whether infected or not. It will come as no surprise to those practicing adolescent medicine that this age group remains below the national average for viral load suppression (a major goal of HIV treatment), particularly in youth with unstable housing. Although challenges related to endemic poverty remain, these safety net programs should be viewed as a success story for a chronic illness that disproportionally impacts impoverished people. While beyond the scope of this article, many providers today are advocating for similar federal programs to address the opioid epidemic that is also inextricably linked to the problem of poverty.

We advocate for continued funding, surveillance, and research into quality improvement of services that address poverty and chronic illness as family and community needs evolve. Many of the programs our patients rely on a routine basis are at significant risk for serious cuts in funding. We must lobby for continued support to ensure that safety net programs for our most vulnerable patients survive despite the constant threats. Moreover, a federal declaration to eliminate poverty in the United States, with appropriate resources dedicated to this cause, would be the bold vision needed to fully address its negative health consequences. Facing the real problems associated with income inequality and prioritizing tools to close gaps between the rich and poor would be a positive step towards ending health disparities that contribute to chronic diseases of childhood. We call for a plan, similar to the United Nations Sustainable Development Goals (with goals $1-3$ being no poverty, zero hunger, and good health and well-being), in which the United States develops a framework for agencies to promote access to the wealth and opportunity of our country for all children and families. This framework would be expected to include benchmarks for success, accountability metrics, and action plans for realtime improvements to implementation. Significant reductions in poverty-related chronic illnesses can be achieved by translating research into policy that incites measurable change for communities.

\section{ADDITIONAL INFORMATION}

Competing interests: The authors declare no competing interests.

Publisher's note: Springer Nature remains neutral with regard to jurisdictional claims in published maps and institutional affiliations.

\section{REFERENCES}

1. Kochmanski, J., Goodrich, J. M., Peterson, K. E., Lumeng, J. C. \& Dolinoy, D. C. Neonatal bloodspot DNA methylation patterns are associated with childhood weight status in the Healthy Families Project. Pediatr. Res. 48, 1514-1522 (2018)

2. Oliveira F. M. S., et al. Abnormalities in autonomic function in obese boys at-risk for insulin resistance and obstructive sleep apnea. Pediatr Res. 2018. [Epub ahead of print].

3. Monthé-Drèze C., Rifas-Shiman S. L., Gold D. R., Oken E., Sarbattama S. Maternal obesity and offspring cognition: the role of inflammation. Pediatr Res. 2018. [Epub ahead of print].

4. WHO. Guideline: Assessing and Managing Children at Primary Health-Care Facilities to Prevent Overweight and Obesity in the Context of the Double Burden of Malnutrition: Updates for the Integrated Management of Childhood Illness (IMCI). World Health Organization, Geneva, 2017. https://www.ncbi.nlm.nih.gov/ books/NBK487902/. Accessed 9 January 2019.

5. Fontenot, Kayla, Semega, Jessica \& Kollar, Melissa U.S. Census Bureau, Current Population Reports, P60-263, Income and Poverty in the United States: 2017. (U.S. Government Printing Office, Washington, DC, 2018).

6. Pascoe J.M., \& Wood D.L. \& Duffee, J.H. \& Kuo A. Committee on psychosocial aspects of child and family health; Council on community pediatrics Mediators and adverse effects of child poverty in the United States. Pediatrics 137, e20160340 (2016).

7. Centers for Medicare \& Medicaid Services. October 2018 Medicaid \& CHIP Enrollment Data Highlights. https://www.medicaid.gov/medicaid/programinformation/medicaid-and-chip-enrollment-data/report-highlights/index.html. Accessed 9 January 2019.

8. Wagnerman K., Chester, A. and Alker, J. Medicaid Is A Smart Investment in Children. Georgetown University Health Policy Institute: Center for Children and Families: Georgetown University Health Policy Institute. 2017. https://ccf. georgetown.edu/wp-content/uploads/2017/03/MedicaidSmartInvestment.pdf. Accessed 9 January 2019.

9. Boudreaux, M. H., Golberstein, E. \& McAlpine, D. D. "The long-term impacts of medicaid exposure in early childhood: evidence from the program's origin". J. Health Econ. 45, 161-175 (2016).

10. Wherry L., et al. Childhood medicaid coverage and later life health care utilization. National Bureau of Economic Research. 2015. https://www.nber.org/papers/ w20929. Accessed 9 January 2019.

11. Sommers $\mathrm{BDaO}, \mathrm{D}$. The poverty-reducing effect of Medicaid. J. Health Econ. 32, 816-832 (2013).

12. Cohodes S.R., \& Grossman D.S. \& Kleiner S.A. \& Lovenheim M.F. The effect of child health insurance access on schooling: evidence from public insurance expansions. J. Hum. Res. 51, 727-759 (2016).

13. Council on community pediatrics. Poverty and Child Health in the United States. Pediatrics. 137, e20160339 (2016).

14. Hess, K. L., Hu, X., Lansky, A., Mermin, J. \& Hall, H. I. Lifetime risk of a diagnosis of HIV infection in the United States. Ann. Epidemiol. 27, 238-243 (2017). 